\title{
Near-Infrared-Fluorescent Probes for Bioapplications based on Silica-Coated Gold Nanobipyramids with Distance-Dependent Plasmon-Enhanced Fluorescence
}

Caixia Niu, ${ }^{\dagger}$ Quanwei Song, ${ }^{, / S}$ Gen He, ${ }^{\dagger} \mathrm{Na} \mathrm{Na},{ }^{\dagger}$ and Jin Ouyang ${ }^{*,{ }^{\prime}}$

†Key Laboratory of Theoretical and Computational Photochemistry, Ministry of Education, College of Chemistry, Beijing Normal University, Beijing 100875, China ${ }^{\ddagger}$ State Key Laboratory of Petroleum Pollution Control, Beijing, 102206

${ }^{\S}$ CNPC Research Institute of Safety and Environmental Technology, Beijing, 102206

*Jin Ouyang Fax: 86 010-62799838; Tel: 86 010-58805373; E-mail: jinoyang@bnu.edu.cn

\section{Contents}

1. Characterization of supernate $\mathrm{Au} / \mathrm{Ag}$ NRs and $\mathrm{Au} / \mathrm{Ag}$ NPs. (Figure S1)

2. Characterization of Au NBPs. (Figure S2)

3. Chemical structure of Cy7 NHS ester. (Figure S3)

4. Characterization of $\mathrm{SiO}_{2} @ \mathrm{Cy} 7 \mathrm{NPs}$. (Figure S4)

5. Calculation of the relative ratio of Cy7 to Au NBP@ $\mathrm{SiO}_{2}$. (Section S2.)

6. PEF studies of Au NBP@SiO $@$ @Cy7 NPs. (Figure S5)

7. Characterization of Au NBP@ $\mathrm{SiO}_{2}$ NPs. (Figure S6)

8.PEF studies of Au NR@SiO $@ @$ Cy7 NPs. (Figure S7)

9. FDTD simulation of electric field maps of a Au NBP and Au NR. (Figure S8)

10. The selectivity of Au NBP@SiO $@ \mathrm{Sy}_{2}-\mathrm{Cu}^{2+} \mathrm{NPs}$ towards PPi. (Figure S9)

11. MTT assay of HeLa cells treated with Au NBP@SiO $@$ Cy7 NPs. (Figure S10)

12. The oligonucleotides used in this work. (Table S1) 

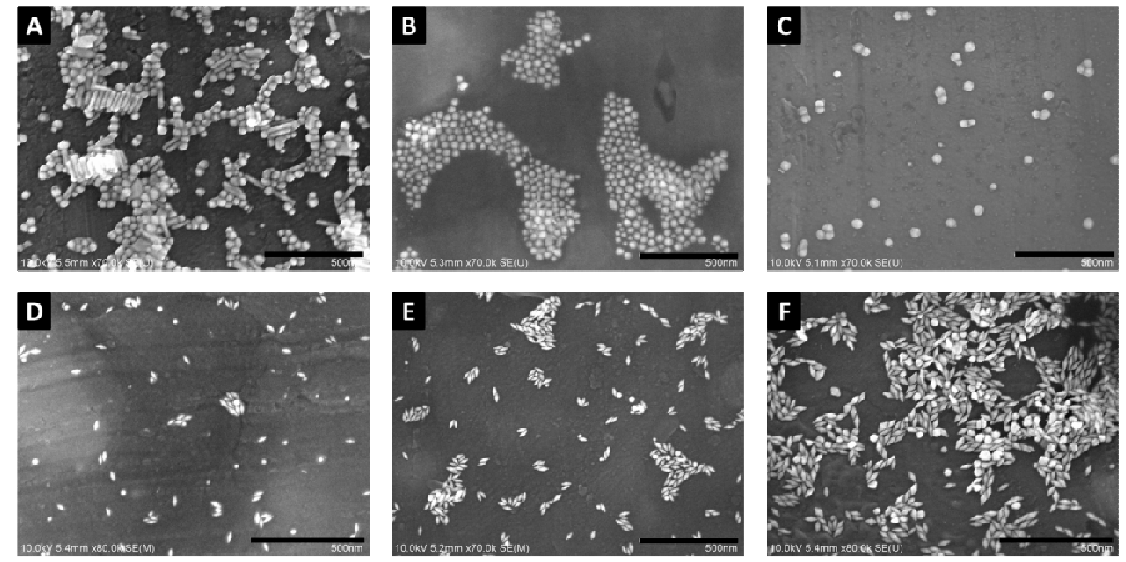

Figure S1. SEM results of supernate $\mathrm{Au} / \mathrm{Ag}$ NRs and $\mathrm{Au} / \mathrm{Ag}$ NPs product with $\mathrm{CTAB}$ of (A) $0.05 \mathrm{M}$, (B) $0.1 \mathrm{M}$ and (C) $0.2 \mathrm{M}$; SEM results of Au NBPs product with CTAB (D) $0.05 \mathrm{M}$, (E) $0.1 \mathrm{M}$ and (F) $0.2 \mathrm{M}$.
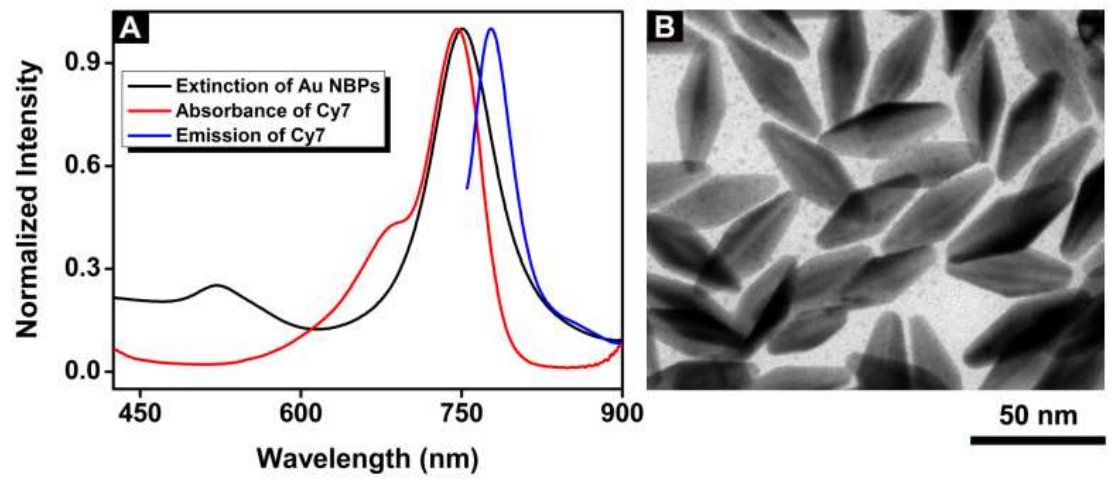

Figure S2. (A) Absorption spectra (red line), emission spectra (blue line) of Cy7 and the extinction spectra of Au NBPs (black line) with LPRW at $750 \mathrm{~nm}$. (B) TEM image of related Au NBPs used in this study with LPRW at $750 \mathrm{~nm}$. 


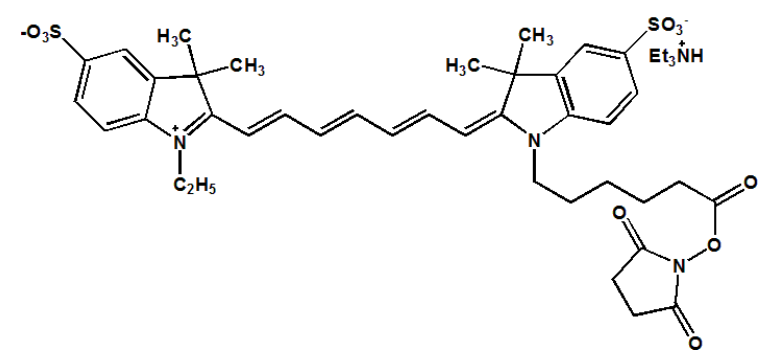

Figure S3. Chemical structure of Cy7 NHS ester.

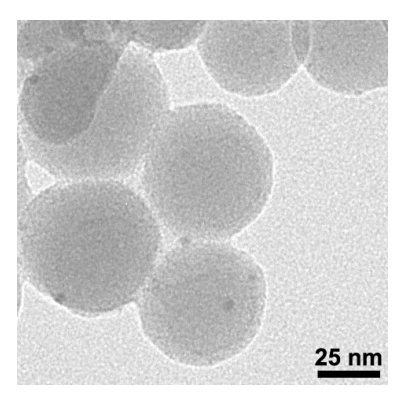

Figure S4. TEM image of $\mathrm{SiO}_{2} @ \mathrm{Cy} 7$ NPs.

Section S2. Calculation of the relative ratio of Cy7 to Au NBP@SiO

The detailed description about the calculation of the relative ratio of $\mathrm{Cy} 7$ to $\mathrm{Au}$ $\mathrm{NBP} @ \mathrm{SiO}_{2}$ are shown below:

The relative ratio of $\mathrm{Cy} 7$ covalently linked to $\mathrm{Au} \mathrm{NBP} @ \mathrm{SiO}_{2}$ could be calculated from the absorption spectra. ${ }^{1-3}$

The used formular is

$$
\begin{aligned}
& N=c \cdot V \cdot N_{A} \\
& A=\varepsilon \cdot b \cdot c \\
& N=\frac{A \cdot V \cdot N_{A}}{\varepsilon \cdot b}
\end{aligned}
$$

a. The number of Cy7 molecules covalently linked to Au NBP@ $\mathrm{SiO}_{2} \mathrm{NPs}\left(N_{\mathrm{Cy} 7}\right)$ :

$$
\begin{aligned}
& N_{\mathrm{Cy} 7}=N_{\mathrm{Cy} 7 \text {-original }}-N_{\mathrm{Cy} 7-\text { supernatant }} \\
& =\frac{\left(A_{\mathrm{Cy} 7 \text {-original }} \cdot V_{\mathrm{Cy} 7-\text { original }}-A_{\mathrm{Cy} 7 \text {-supernatant }} \cdot V_{\mathrm{Cy} 7 \text {-supernatant }}\right) \cdot N_{A}}{\varepsilon_{C y 7} \cdot b}
\end{aligned}
$$


b. The number of Au NBP NPs in the solution for linking Cy7 molecules $\left(N_{\mathrm{NP}}\right)$ :

$$
N_{N P}=\frac{A_{N P} \cdot V_{N P} \cdot N_{A}}{\varepsilon_{N P} \cdot b}
$$

c. Therefore, the relative ratio of $\mathrm{Cy} 7$ to $\mathrm{Au} \mathrm{NBP} @ \mathrm{SiO}_{2}$

$=$ The number of conjugated Cy7 molecules/The number of Au NBP NPs $\left(\mathrm{N}_{\mathrm{Cy}} / \mathrm{N}_{\mathrm{NP}}\right)$ :

$$
\frac{N_{C y z}}{N_{N p}}=\frac{\left(A_{C y 7 \text { ariginal }} \cdot V_{C y 7 \text { ariginal }}-A_{C y 7 \text { supernatant }} \cdot V_{C y 7 \text { supernatant }}\right) \cdot \varepsilon_{N P}}{A_{N p} \cdot V_{N ?}{ }^{r} \cdot \varepsilon_{C y 7}}
$$

In detail, $A_{\mathrm{Cy} 7}, \varepsilon_{\mathrm{Cy} 7}, A_{\mathrm{NP}}$ and $\varepsilon_{\mathrm{NP}}$ are the absorbance and molar extinction coefficients of $\mathrm{Cy} 7$ and $\mathrm{Au} \mathrm{NBP} @ \mathrm{SiO}_{2}$, respectively. $\varepsilon_{\mathrm{Cy} 7}=1.99 \times 10^{5} \mathrm{M}^{-1} \mathrm{~cm}^{-1}$ and $\varepsilon_{\mathrm{NP}}=2.3 \times 10^{10} \mathrm{M}^{-1} \mathrm{~cm}^{-1}$. The Cy7 NHS ester was purchased from the Tianjin Biolite Biotech Co., Ltd. The value of $\varepsilon_{\mathrm{Cy} 7}$ can be get from the product specifications. The value of $\varepsilon_{\mathrm{NP}}$ was calculated according to the reported works. ${ }^{4}$

Finally, the calculated result is that each Au NBP@SiO $2 @$ Cy7 NP contains 3900 Cy7 molecules.
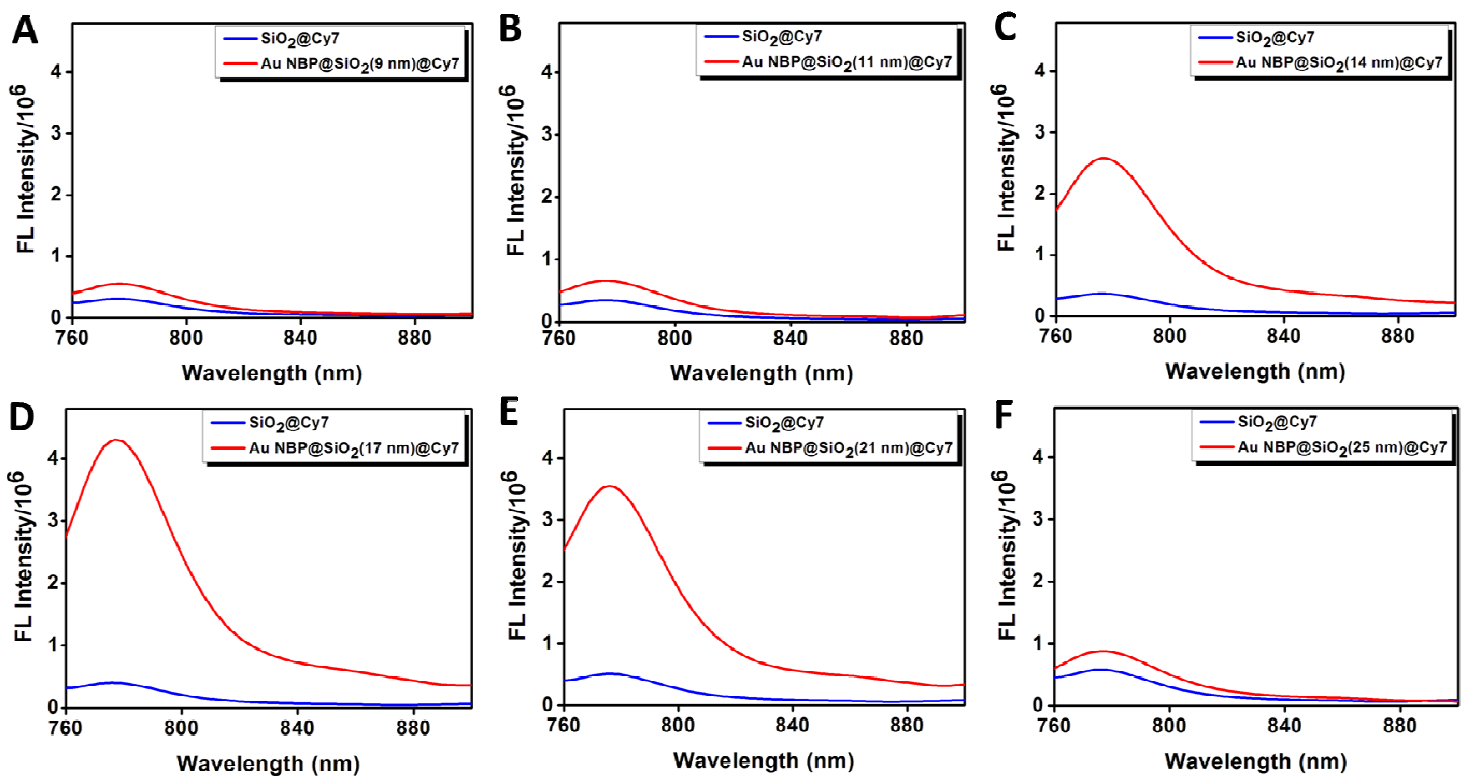

Figure S5. Fluorescence spectra of $\mathrm{SiO}_{2} @ \mathrm{Cy} 7$ and Au NBP@ $\mathrm{SiO}_{2} @ \mathrm{Cy} 7$ with silica 
thickness of (A) $9 \mathrm{~nm}$, (B) $11 \mathrm{~nm}$, (C) $14 \mathrm{~nm}$, (D) $17 \mathrm{~nm}$, (E) $21 \mathrm{~nm}$ and (F) $25 \mathrm{~nm}$.

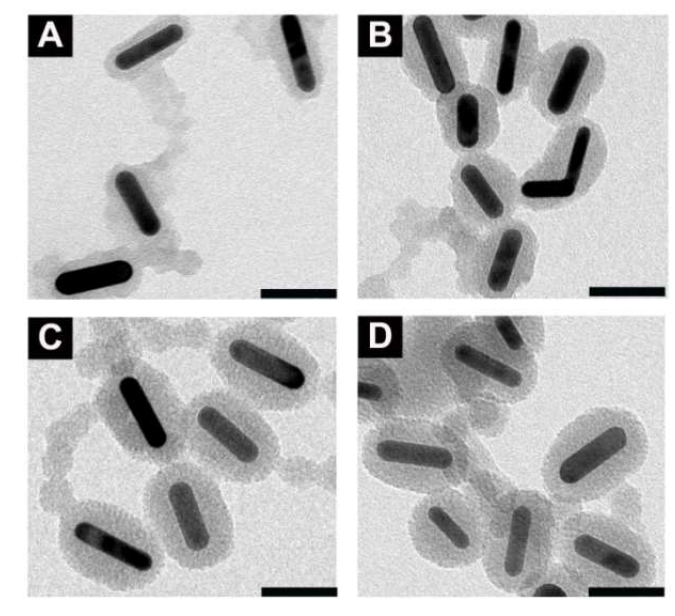

Figure S6. TEM images of silica-coated Au NRs with silica thickness of (A) 9 nm, (B) $14 \mathrm{~nm},(\mathrm{C}) 17 \mathrm{~nm}$ and (D) $21 \mathrm{~nm}$. The scale bars represent $50 \mathrm{~nm}$.
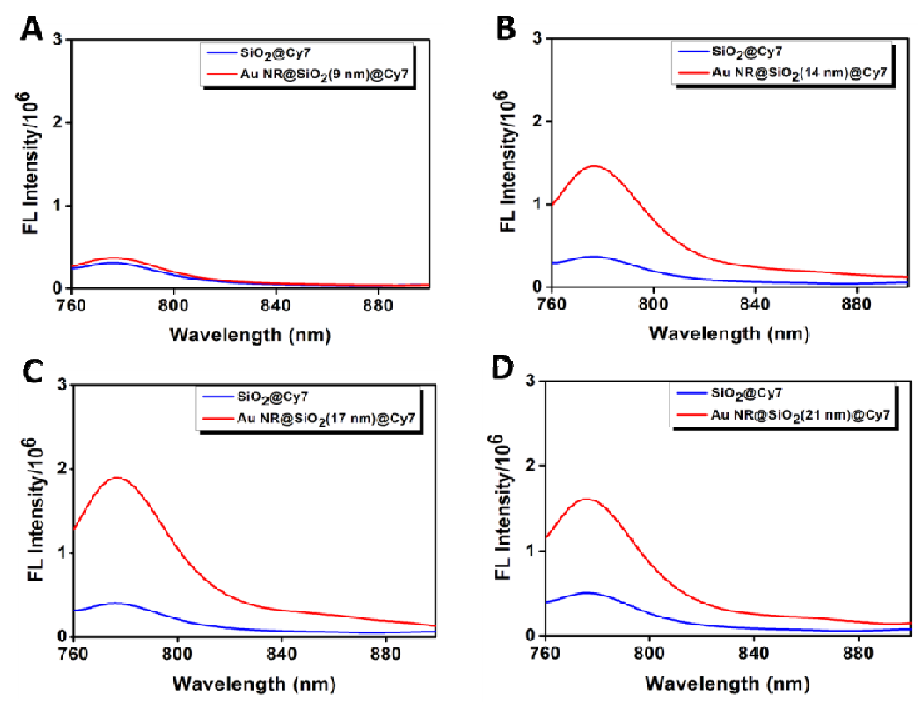

Figure S7. Fluorescence spectra of $\mathrm{SiO}_{2} @ \mathrm{Cy} 7 \mathrm{NPs}$ and $\mathrm{Au} \mathrm{NR} @ \mathrm{SiO}_{2} @ \mathrm{Cy} 7 \mathrm{NPs}$ with silica thicknesses of (A) $9 \mathrm{~nm}$, (B) $14 \mathrm{~nm}$, (C) $17 \mathrm{~nm}$ and (D) $21 \mathrm{~nm} .$. 
A

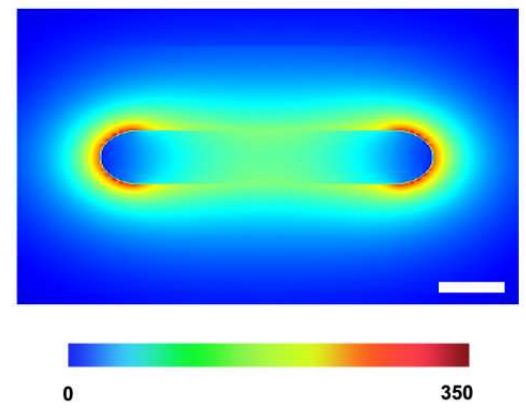

B

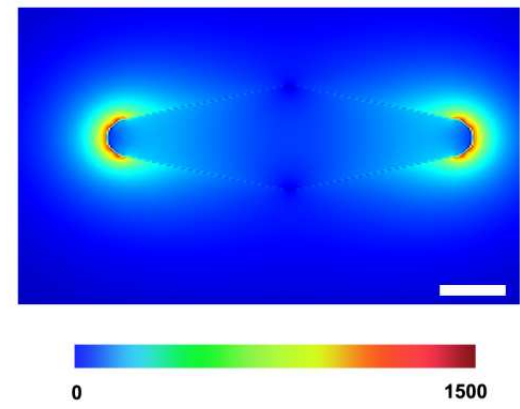

Figure S8. The corresponding computed electric field maps of a (A) Au NBP and (B) Au NR at their LPRW ( $\lambda=750 \mathrm{~nm}$ ), respectively. The scale bar represents $10 \mathrm{~nm}$.

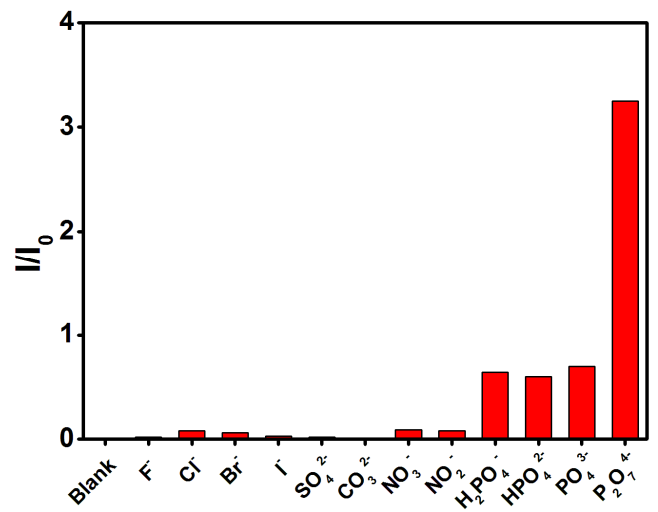

Figure S9. The fluorescence intensity ratios $I / I_{0}$ of $\mathrm{Au} \mathrm{NBP} @ \mathrm{SiO}_{2} @ \mathrm{Cy} 7-\mathrm{Cu}^{2+} \mathrm{NPs}$ with $\mathrm{F}^{-}, \mathrm{Cl}^{-}, \mathrm{Br}^{-}, \mathrm{I}^{-}, \mathrm{SO}_{4}{ }^{2-}, \mathrm{CO}_{3}{ }^{2-}, \mathrm{NO}_{3}{ }^{-}, \mathrm{NO}_{2}{ }^{-}, \mathrm{H}_{2} \mathrm{PO}_{4}^{-}, \mathrm{HPO}_{4}{ }^{2-}, \mathrm{PO}_{4}{ }^{3-}, \mathrm{P}_{2} \mathrm{O}_{7}{ }^{4-}$ at $20 \mu \mathrm{M}$ in HEPES buffer (5 mM, pH 7.4).

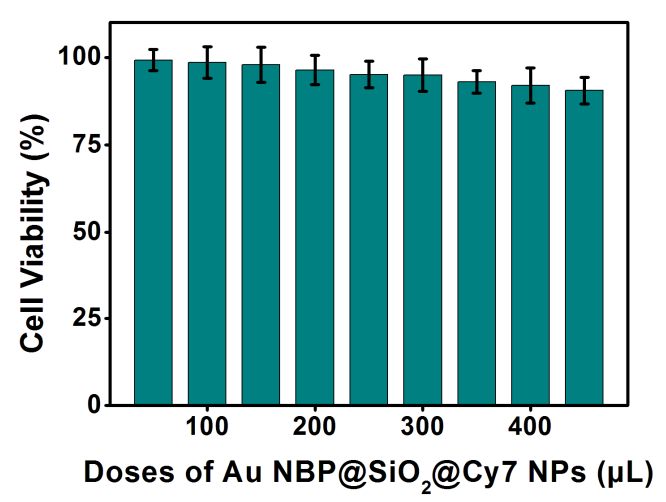

Figure S10. MTT assay of HeLa cells treated with different doses of $\mathrm{Au}$ NBP@SiO $@ @$ Cy7 NPs for 24 h. 
Table S1. The oligonucleotides used for RCA in this work.

\begin{tabular}{l|l}
\hline Probe & Sequence $\left(\mathbf{5}^{\prime} \rightarrow \mathbf{3}\right.$ ') \\
\hline Micro RNA (let-7d) & AGAGGUAGUAGGUUGCAUAGUU \\
\hline Circular template for & TACTCATACGCTCATACGTTCATCACGACTACAACT \\
let-7d & ATGCAACCTACTACCTCTGATCCCCGTTA \\
\hline
\end{tabular}

\section{REFERENCES}

(1) Zhao, T. T.; Yu, K.; Li, L.; Zhang, T. S.; Guan, Z. P.; Gao, N. Y.; Yuan, P. Y.; Li, S. ; Yao, S. Q.; Xu, Q. H.; Xu, G. Q. ACS Appl. Mater. Interfaces 2014, 6, 2700.

(2) Ni, W. H.; Yang, Z.; Chen, H. J.; Li, L.; Wang, J. F. J. Am. Chem. Soc. 2008, 130, 6692.

(3) Lu, L.; Qian, Y. X.; Wang, L. H.; Ma, K. K.; Zhang, Y. D. ACS Appl. Mater. Interfaces 2014, 6, 1944.

(4) Li, Q.; Zhuo, X. L.; Li, S.; Ruan, Q. F.; Xu, Q. H.; Wang, J. F. Adv. Opt. Mater. 2015, 3, 801 . 